\title{
A Cross-Cultural Perspective on Psychological Determinants of Chronic Fatigue Syndrome: a Comparison Between a Portuguese and a Dutch Patient Sample
}

\author{
M. Marques • V. De Gucht • I. Leal • S. Maes
}

(C) International Society of Behavioral Medicine 2012

\begin{abstract}
Background Few studies focus on cross-cultural differences in Chronic fatigue syndrome (CFS).

Purpose This study aimed to (1) compare fatigue severity and impairment, somatic complaints, psychological distress, and quality of life (QoL) in a population of Portuguese and Dutch patients; (2) explore the differential contribution of behavioral and cognitive determinants of fatigue severity; and (3) investigate the relation between fatigue severity and somatic complaints on one hand and QoL on the other in both populations.

Method Eighty-five female patients from Portugal (Mean age= 47.54) and 167 female CFS patients from The Netherlands (Mean age $=44.93$ ) participated in the study. All participants were surveyed for demographic and clinical characteristics, fatigue severity, somatic symptoms, psychological distress, (physical and psychological) QoL, physical activity, behavior regulation patterns, and illness representations.

Results Cross-cultural differences were found in relation to working status, duration of fatigue symptoms, psychological distress, somatic complaints, and psychological QoL. Although behavioral characteristics and illness representations were significantly associated with fatigue severity in both Portuguese and Dutch patients, there were important differences in the determinants of CFS. Moreover, higher levels
\end{abstract}

M. Marques $\cdot$ V. De Gucht $\cdot$ S. Maes

Health Psychology, Leiden University,

Wassenaarseweg 52, P.O. BOX 955, 2300 RB Leiden,

The Netherlands

M. Marques $(\square) \cdot$ I. Leal

Psychology and Health Research Unit (UIPES),

ISPA-University Institute,

Rua Jardim do Tabaco, 34,

1149-041 Lisbon, Portugal

e-mail: mmarques@ispa.pt of fatigue and severity of other somatic complaints were related to poor QoL.

Conclusions These findings show cross-cultural similarities and differences in clinical characteristics and psychological determinants of CFS that are important in view of diagnosis and treatment.

Keywords Chronic fatigue syndrome - Fatigue severity · Psychological determinants $\cdot$ Cross-cultural

\section{Introduction}

Somatic symptoms, such as fatigue, are an important reason for doctor visits $[1,2]$ and it is estimated that these symptoms remain medically unexplained in about one third of the cases [1]. Usually, fatigue is explained by life circumstances and is transitory, but for some, fatigue symptoms are indeed medically unexplained, can be severe and become chronic, resulting in functional and social impairment (e.g., inability to work), high use of health care resources, and lower quality of life (QoL) [2-4]. According to the Centers for Disease Control and Prevention (CDC) fatigue is classified as chronic fatigue syndrome (CFS) when it lasts for at least 6 months, is not alleviated by rest, is debilitating, results in a significant reduction of daily activities, cannot be explained by an organic disease, and is accompanied by four or more of the following symptoms: unrefreshing sleep, lengthy malaise after exertion (lasting for over $24 \mathrm{~h}$ ), impaired memory or concentration, sore throat, tender cervical or axillary lymph nodes, muscle pain, multi-joint pain without swelling or redness, and headaches of a new type or severity [5]. Comorbidity between CFS and psychological distress (depression and anxiety) was found in several studies, although the relationship remains unclear [6-8]. Some prospective studies found the occurrence of 
psychiatric disorders during adulthood to be associated with later CFS $[9,10]$. Other studies suggest that the high rates of psychological distress in CFS patients may be due to a common negative affective reaction to a chronic health problem [6], to disability associated with fatigue [7], to difficulties in the diagnosis of CFS [8], or as a result of the lack of legitimization of the disease by medical doctors [8] among other explanations. Patients with CFS also experience a great number and higher severity of other somatic complaints $[4,11]$. Several studies indicate that these patients are hypersensitive to somatic sensations, which can lead to a worsening and increase of the number of symptoms perceived $[6,12,13]$.

A psychological perspective on CFS can provide a better understanding of the cognitive and behavioral factors associated with the onset and perpetuation of unexplained fatigue $[2$, 6]. More specifically, patients' illness representations and coping strategies can contribute to the worsening and perpetuating of fatigue $[6,13,14]$. Patients with CFS tend to believe that their illness has severe consequences and will last for a long period of time (timeline), and they also expect many associated symptoms $[6,13,15]$. A prospective study revealed that perceived consequences, timeline, uncontrollability, and emotional response predicted worsening and maintenance of chronic fatigue [16]. Patients who belief that they suffer from a severe illness, that their CFS is out of control or incurable and will lead to adverse consequences, usually develop passive ways of coping with their illness, leading to high disability and psychological distress $[2,13,17]$. In relation to behavior regulation (coping), it has been suggested that CFS patients tend to adopt a "boom-and-bust pattern" (also called "all-or-nothing behavior") or a limiting behavior pattern to deal with their illness $[13,16]$. All-or-nothing behavior refers to the systematic alternation between periods of excessive activity (when feeling good), and, as a consequence of that, feeling extremely fatigued and having to rest for longer periods of time [13, 18]. Limiting behavior refers to the excessive resting and inactivity, which can be associated with patient complaints related to exercise intolerance and post-exertional malaise. Patients' perceptions and expectations related to symptom exacerbation as a consequence of exercise can explain the reduced levels of physical activity found in these patients [6]. At the same time, lack of physical activity and excessive resting are factors that can result in physical deconditioning which, in turn, might perpetuate fatigue and physical disability [19]. The importance of identifying and understanding the specific cognitive and behavioral determinants of CFS is reinforced by the promising results of CBT and graded exercise approaches in CFS management [20, 21].

CFS is considered to be a heterogeneous clinical condition. Patients may present with different levels of (mental and physical) fatigue severity, psychological distress, additional somatic symptoms, and different levels of impairment and disability $[3,6]$. For this reason, fatigue severity and related somatic symptoms, psychological distress and QoL, may vary between countries and cultures [22, 23]. Most studies on the prevalence and clinical characteristics of CFS have been conducted in northwestern Europe, North America, and Oceania [23], while there are very few international studies that compare patient populations from different countries [3, 22, 23]. Hickie and colleagues [22] explored CFS worldwide, using existing data from different cultures. Results revealed a fivefactor model of symptoms (musculoskeletal pain and prolonged fatigue, neurocognitive difficulties, sleep disturbance, inflammation, and mood disturbance), confirming that CFS is indeed a universal disease. Another study on health-related QoL conducted with US, UK, and German CFS patients showed that these patients reported a lower QoL in all countries [3]. In a study comparing the prevalence and recognition of CFS in primary health care services between Brazil and England [23], the prevalence of CFS appeared to be similar in both countries, but there were differences in the recognition of CFS as a discrete disorder leading to a lower number of diagnosed patients in Brazil.

Likewise, cross-cultural research on the psychological determinants of CFS, such as behavioral (e.g., physical activity) and cognitive (e.g., illness representations) factors, is lacking, although this type of research can contribute to the cultural adaptation of existing CFS models and the development of tailored treatment strategies. For this reason, we conducted a comparative study between two economically and culturally distinct countries: Portugal (a southern European country) and The Netherlands (a northwestern European country), as we expected differences in clinical characteristics and determinants of CFS. Available data reveal differences in psychological distress and physical activity between the two populations [24, 25].

This is an exploratory study aiming at (1) comparing fatigue impairment and severity, somatic complaints, psychological distress and (physical and psychological) QoL in Portuguese and Dutch CFS patients; (2) exploring differential effects of behavioral (physical activity and behavior regulation patterns) and cognitive factors (illness representations) on fatigue severity in the study populations; and (3) examining the contribution of fatigue severity and somatic complaints to (physical and psychological) QoL in Portuguese and Dutch CFS patients.

\section{Method}

Participants and Procedures

This cross-sectional study included participants from two countries: Portugal and The Netherlands. In both cases, inclusion criteria were the following: (1) meeting the CDC 
criteria for CFS [5]; (2) being at least 18 years old; (3) being fluent in Portuguese/Dutch; (4) and having the capacity to provide an informed consent. Exclusion criteria were similar for both samples: presence of a concurrent somatic condition that could explain the fatigue symptoms and/or presence of a severe psychiatric disorder (according to the CDC criteria for exclusionary medical and psychiatric conditions). Table 1 presents the demographic and clinical characteristics of the samples.

Participants from Portugal were recruited via various health care institutions and from the National Chronic Fatigue Syndrome and Fibromyalgia Patients Association. CFS patients from the health care institutions were referred by their medical doctor based on the inclusion and exclusion criteria for the study. Patients from the patients association had a clinical diagnosis of unexplained chronic fatigue. Patients were approached by the research team to complete the questionnaires. Participants from The Netherlands were recruited through the National Chronic Fatigue Syndrome Association. All Dutch patients were medically diagnosed as having CFS. The members of this association were invited via e-mail by the patient association to complete the questionnaire. For both samples, informed consent was obtained, and confidentiality of the data was guaranteed by the research team. Furthermore, the inclusion and exclusion criteria were checked by the research team, using self-report measures (CDC checklist of CFS symptoms; presence and name of chronic disease; and presence and name of psychiatric disorder)

For this study, patients from Portugal and The Netherlands were matched on age, gender (only female patients were included), and diagnosis (only patients fulfilling the CDC criteria for CFS were included).
Table 1 Descriptives and differences in demographics and clinical characteristics, fatigue severity, somatic complaints, psychological distress and QoL for the Portuguese and the Dutch CFS samples
Values are the mean \pm SD unless otherwise indicated

${ }^{*} p<0.05 ; * * p<0.01$

\begin{tabular}{|c|c|c|c|}
\hline & $\begin{array}{l}\text { Portugal } \\
(N=85)\end{array}$ & $\begin{array}{l}\text { Netherlands } \\
(N=167)\end{array}$ & $\begin{array}{l}\text { Test for } \\
\text { differences }\end{array}$ \\
\hline Female patients, no. (\%) & 100 & 100 & - \\
\hline Age, years & $47.54 \pm 10.75$ & $44.93 \pm 10.98$ & $t=1.77$ \\
\hline Educational level, no. (\%) & & & $X^{2}=7.02 *$ \\
\hline Primary and Lower education & 27.4 & 16.2 & \\
\hline Secondary education & 39.3 & 34.7 & \\
\hline Tertiary education & 33.3 & 49.1 & \\
\hline Not working, no. (\%) & 44.7 & 68.7 & $X^{2}=13.5^{* *}$ \\
\hline Illness duration, months & $128.81 \pm 102.41$ & $165.71 \pm 92.22$ & $t=-2.85^{* *}$ \\
\hline Medical visits, no. & $4.64 \pm 3.42$ & $4.28 \pm 4.67$ & $t=0.59$ \\
\hline CDC CFS Diagnosis, no. (\%) & 100 & 100 & - \\
\hline Fatigue severity & $101.75 \pm 17.60$ & $99.52 \pm 16.61$ & $t=0.99$ \\
\hline Subjective experience of fatigue & $46.73 \pm 6.88$ & $46.34 \pm 7.96$ & $t=0.38$ \\
\hline Subjective experience of fatigue, no. (\%) & & & $X^{2}=0.47$ \\
\hline Non clinical & 7.1 & 9.6 & \\
\hline Clinical & 92.9 & 90.4 & \\
\hline Somatic complaints & $15.53 \pm 4.11$ & $14.29 \pm 4.24$ & $t=2.20^{*}$ \\
\hline Somatic complaints, no.(\%) & & & $X^{2}=3.51$ \\
\hline Low & 7.1 & 13.5 & \\
\hline Medium & 42.4 & 46.0 & \\
\hline High & 50.6 & 40.5 & \\
\hline Depression & $1.72 \pm 0.92$ & $0.84 \pm 0.78$ & $t=7.61 * *$ \\
\hline Depression, no. (\%) & & & $X^{2}=53.09 * *$ \\
\hline Non clinical & 48.2 & 89.8 & \\
\hline Clinical & 51.8 & 10.2 & \\
\hline Anxiety & $1.67 \pm 0.79$ & $0.79 \pm 0.77$ & $t=8.42 * *$ \\
\hline Anxiety, no. (\%) & & & $X^{2}=39.83^{* *}$ \\
\hline Non clinical & 35.3 & 76.0 & \\
\hline Clinical & 64.7 & 24.0 & \\
\hline Physical QoL & $37.22 \pm 18.40$ & $34.83 \pm 16.91$ & $t=1.03$ \\
\hline Psychological QoL & $39.31 \pm 16.97$ & $45.26 \pm 9.22$ & $t=-3.61^{* *}$ \\
\hline
\end{tabular}


Measures

\section{Patient Characteristics}

Sociodemographic characteristics include age, gender, education, and employment status (Table 1). Clinical information was gathered using four indicators: (1) presence of persistent fatigue, (2) duration of fatigue symptoms (months), (3) number of doctor visits in the previous 6 months, and (4) a CDCbased symptom checklist for CFS. The checklist includes the eight major symptoms of CFS defined by the CDC criteria [5]. Respondents are asked to rate using a dichotomous scale (yes/ no) whether they experienced the symptoms for the last 6 months. To be diagnosed with CFS, patients need to have a complaint of persistent unexplained fatigue for at least 6 months and have at least four of the major CFS symptoms listed by the CDC [5].

\section{Fatigue Severity}

The Checklist of Individual Strength (CIS-20R) was used to assess fatigue levels [26]. The CIS-20R is a 20-item self-report questionnaire that assesses four dimensions of fatigue: subjective experience of fatigue, lack of concentration, lack of motivation, and activity reduction. Items are rated on a 7-point Likert scale ranging from "Yes, that is true" to "No, that is not true". A total CIS-20R score (fatigue severity), ranging from 20 to 140, can be calculated by adding the scores from each dimension. Higher scores indicate higher levels of fatigue severity. For the purpose of this study, only the total fatigue severity score and the subjective experience of fatigue dimension were used. A cut-off point of 35 for subjective experience of fatigue [27] is used to define clinical levels of fatigue. The CIS-20R is a well validated and reliable measure for CFS patients [26]. Data on the Portuguese version of the CIS-20R reveal good reliability of the dimensions subjective experience of fatigue and total fatigue severity $(\alpha=0.89$ and $\alpha=0.90$, respectively) [28].

\section{Somatic Complaints}

Severity of physical symptoms was measured by means of the Patient Health Questionnaire-15 (PHQ-15) [1]. The PHQ-15 assesses the presence and severity of 15 somatic symptoms (e.g., back pain). A higher score indicates a higher level of somatization. Moreover, scores of 5, 10, and 15 represent cutoff points for low, medium, and high somatic symptom severity, respectively [1].

\section{Psychological Distress}

The depression and anxiety subscales from the validated Brief Symptom Inventory (BSI) were used to assess levels of psychological distress [29]. Individuals rank each symptom on a 5-point Likert scale (from "never" to "very frequently"). Final scores range from 0 to 4 and higher scores represent higher levels of depression and anxiety. Cut-off points of 1.80 and 1.33 are used to define clinical levels of depression and anxiety, respectively [30].

\section{Quality of Life}

The Short Form Health Survey-12 (SF-12 v2) [31] was used to assess physical and psychological functioning and overall health-related QoL. The SF-12v2 is a well validated measure consisting of eight domains: (1) general health perception, (2) physical functioning, (3) role limitations due to physical problems, (4) bodily pain, (5) vitality, (6) role limitations due to emotional problems, (7) social functioning, (8) and mental health. These eight dimensions are combined into a physical functioning score (physical QoL) and a psychological functioning score (psychological QoL), ranging from 0 to 100, with lower scores representing worst health functioning and QoL.

\section{Physical Activity}

Levels of physical activity were assessed using the Short Questionnaire to Assess Health-Enhancing Physical Activity [32] sports section in which participants indicate the types of physical activities they presently do (e.g., swimming), the frequency per week (e.g., 3 days per week), and duration per day (e.g., $50 \mathrm{~min}$ ). Bicycling and walking frequency were also included. For the purpose of this study, the intensity of each activity was not included. To score the physical activity measure, total minutes of activity is calculated for each activity by multiplying frequency (days/week) and duration (minutes/day). Total physical activity score for each participant was calculated by making the sum of each activity score.

\section{Behavior Regulation Patterns}

Using the all-or-nothing and limiting behavior scales from The Behavioral Responses to Illness Questionnaire (BRIQ), behavior regulation patterns were assessed [33]. The first dimension assesses the "boom-and-bust pattern" usually observed in CFS, and the second dimension assesses the limitations in daily activities and the excessive rest that patients take due to their fatigue problems. Higher scores (ranging from 1 to 5) represent a more frequent use of a boom-and-bust pattern and more reduced daily activities, respectively. Portuguese and Dutch versions of the BRIQ have a very good internal consistency for the all-or-nothing scale $(\alpha=0.84$ and $\alpha=0.88$, respectively), but much lower, although still acceptable alphas, for the limiting behavior scale $(0.68$ and 0.72 , respectively). 


\section{Patients' Illness Representations}

Using the Brief Illness Perception Questionnaire (brief IPQ), the patients' illness representations were assessed [34]. The brief IPQ consists of eight items, rated on a 10-point scale, representing eight illness perceptions: illness consequences, timeline (expected duration of illness), personal control, treatment control, identity (disease label), illness coherence (understanding), illness concern, and emotional response. The brief IPQ dimensions are scored separately. Higher scores on illness consequences, timeline, identity, illness concern, and emotional response indicate a higher perceived impact of CFS. Higher scores on personal control, treatment control, and illness coherence, represent more positive illness representations or, in other words, control over the illness.

\section{Data Analyses}

Descriptive data and differences between the Portuguese and Dutch samples for demographics and clinical information related to CFS were explored using univariate chi-square tests (for dichotomous variables) and two-sided $t$ tests (for continuous variables). Pearson correlation coefficients were conducted to examine the univariate relations between the determinants and the dependent variables (fatigue severity, and physical and psychological QoL). For statistical power reasons, only determinants that showed a significant relation with the dependent variables at a $p<0.01$ level in either sample were entered in the subsequent hierarchical regression analyses (Tables 2 and 3). Therefore, the regression analyses examined the respective contribution of the behavioral characteristics (physical activity, all-or-nothing behavior, and limiting behavior) (block 1) and the illness representations consequences, personal control, identity, illness concern, and emotional response (block 2) to fatigue severity (Table 4). In addition, regression analyses were conducted to examine the association between fatigue severity and somatic complaints on one hand and physical and psychological QoL on the other hand. There was no multicollinearity between the variables. For the regression analyses, we considered $p$ values lower than or equal to 0.05 as significant. Data analyses were conducted using the statistical software SPSS v19.

\section{Results}

Descriptive and Univariate Analyses

Table 1 presents descriptive data for both study populations and the results of comparative tests for the following variables: demographic characteristics, working status, use of health care resources, fatigue symptoms, fatigue severity, somatic complaints, psychological distress, and QoL (physical and psychological functioning) in Portugal and The Netherlands. A significant difference was found for educational level $(p<0$. $05)$. Dutch patients had a higher educational level than Portuguese patients. Furthermore, there was a significant difference in working status: $68.7 \%$ of the Dutch respondents reported not to be working, while $44.7 \%$ of the Portuguese reported not to work. In addition, there was a longer duration of fatigue symptoms $(p<0.01)$ in the Dutch sample (13.8 years) than in the Portuguese sample (10.7 years).

No significant differences were found for fatigue severity and subjective experience of fatigue. Both Portuguese and Dutch patients presented high levels of fatigue severity and subjective experience of fatigue. The large majority of the patients in both samples met clinical levels of fatigue. Portuguese patients reported a higher severity of (other) somatic symptoms than Dutch patients $(p<0.05)$. In relation to psychological distress, the Portuguese CFS patients reported significantly higher levels of depression $(p<0.001)$ and anxiety $(p<$ 0.001 ), while significantly, more Portuguese participants also reached a clinical level of depression ( 51.8 vs $10.2 \% ; p<0.001)$ and anxiety ( 64.7 vs $24 \% ; p<0.001)$. Both Portuguese and Dutch patients presented low levels of physical and psychological functioning, but the score for psychological functioning was significantly higher in the Dutch population $(p<0.01)$.

\section{Hierarchical Regression Analyses}

Table 4 shows the results of the hierarchical regression models for fatigue severity. In the Portuguese sample, the regression model including the behavioral factors (block 1$)$ was significantly different from the null model $(F(3)=10.022, p<0.001)$ and explained $27 \%$ of the variance in fatigue severity. Higher levels of physical activity were significantly associated with lower fatigue severity $(p<0.001)$. In addition, patients who importantly reduced their daily activities (limiting behavior) presented higher fatigue severity levels $(p<0.05)$. Adding illness representations to the model led to an increase of $18 \%$ in explained variance (block 2). Higher identity beliefs and higher emotional response were significantly associated with higher fatigue severity levels $(p<0.05$ and $p<0.01$, respectively). The final model explained $45 \%$ of the variance in fatigue severity $(F(2)=7.848, p<0.001)$. With respect to the fatigue severity in the Dutch sample, the regression model including the behavioral factors (block I) was also significantly different from the null model $(F(2)=11.644, p<0.001)$ and explained $18 \%$ of the variance in fatigue severity. Patients who adopted an all-or-nothing behavior pattern presented higher fatigue severity levels $(p<0.05)$. Illness representations (block 2) led to a significant increase in the explained variance $(27 \%)$. A higher belief in personal control over CFS was significantly associated with lower levels of fatigue severity $(p<0.001)$. Furthermore, patients who considered the illness as threatening (consequences) 


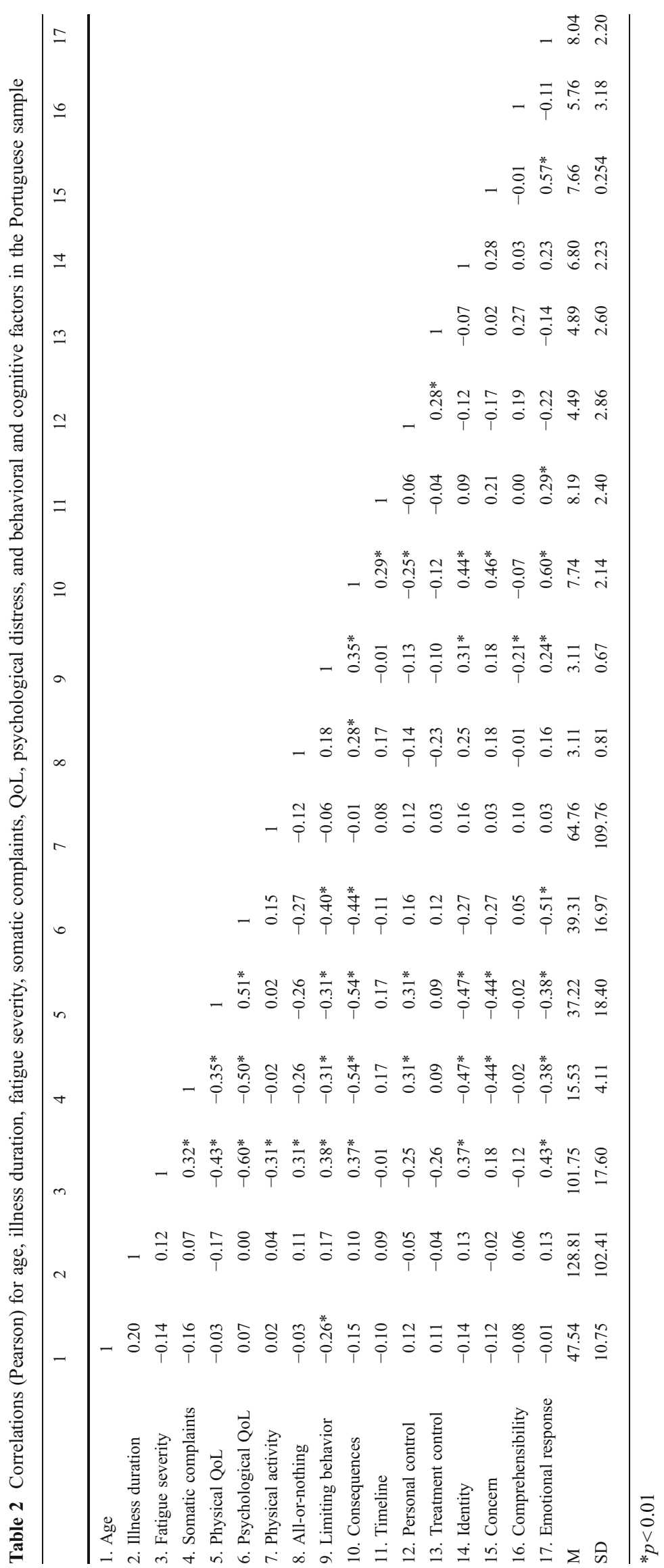




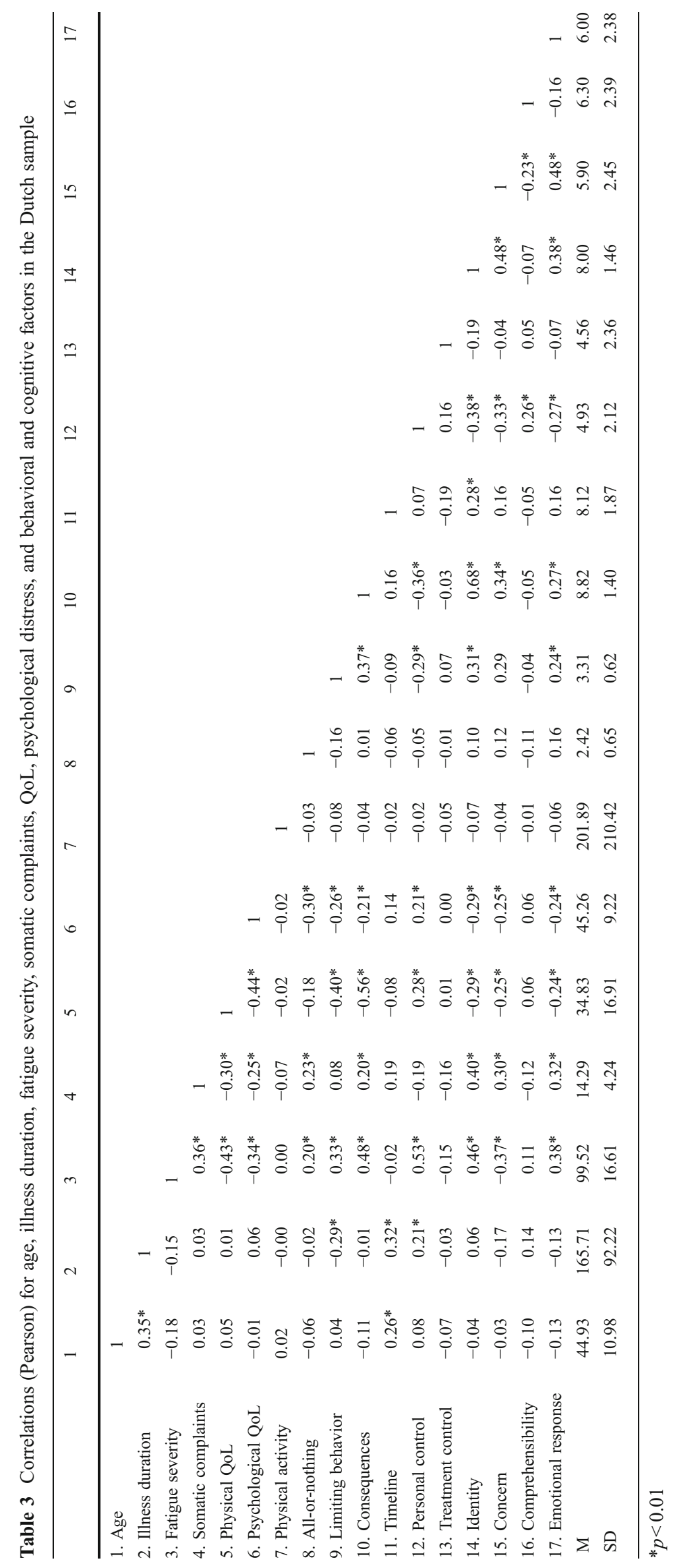


Table 4 Hierarchical regression analyses for fatigue severity by country

\begin{tabular}{|c|c|c|c|c|}
\hline \multirow[b]{3}{*}{ Predictors } & \multicolumn{4}{|c|}{ Fatigue severity } \\
\hline & \multicolumn{2}{|c|}{ Portugal } & \multicolumn{2}{|c|}{ Netherlands } \\
\hline & $\Delta R^{2}$ & $\beta$ & $\Delta R^{2}$ & $\beta$ \\
\hline Block 1 (behavioral factors) & $0.27 * *$ & & $0.18 * *$ & \\
\hline Physical activity & & $-31^{* *}$ & & 0.03 \\
\hline All-or-nothing behavior & & 0.13 & & $0.16^{*}$ \\
\hline Limiting behavior & & $0.20 *$ & & 0.12 \\
\hline $\begin{array}{l}\text { Block } 4 \text { (Illness } \\
\text { representations) }\end{array}$ & $0.18^{* *}$ & & $0.27 * *$ & \\
\hline Consequences & & -0.03 & & $0.22 * *$ \\
\hline Personal control & & -0.09 & & $-0.34 * *$ \\
\hline Identity & & $0.25^{*}$ & & 0.08 \\
\hline Concern & & -0.16 & & 0.03 \\
\hline Emotional response & & $0.38 * *$ & & 0.14 \\
\hline$R^{2}$ & 0.45 & & 0.46 & \\
\hline Adj. $R^{2}$ Model & $0.40^{* *}$ & & $0.43^{* *}$ & \\
\hline
\end{tabular}

Standardized beta coefficients $(\beta)$ represent the beta at final entry $* p<0.05 ; * * p<0.01$

presented higher fatigue severity levels $(p<0.01)$. The final model explained $46 \%$ of the variance in fatigue severity $(F(8)=16.287, p<0.001)$.

The regression model for physical QoL including fatigue severity and somatic complaints was significantly different from the null model, in both the Portuguese $(F(2)=12.262$, $p<0.001)$ and the Dutch $(F(2) 22.71, p<0.001)$ sample, explaining 23 and $22 \%$, respectively, of the variance in this variable. Higher fatigue severity and somatic distress were significantly associated with lower levels of physical functioning in Portuguese $(\beta=-0.39, p<0.001$ and $\beta=-0.23, p<$ 0.05 , respectively) and Dutch patients $(\beta=-0.17, p<0.05$ and $\beta=-0.19, p<0.05$, respectively). Similar results were found for psychological QoL. The regression models including fatigue severity and somatic complaints were significantly different from the null model in both the Portuguese $(F(2)=$ 25.207, $p<0.001)$ and the Dutch sample $(F(2)=12.727, p<$ 0.001 ), explaining 46 and $14 \%$, respectively, of the variance in psychological QoL. Higher fatigue severity was significantly associated with lower levels of psychological functioning in both samples $(\beta=-0.49, p<0.001$ and $\beta=-0.29, p<0.001$, respectively). In the Portuguese sample, a higher level of somatic distress was also significantly associated with lower psychological QoL $(\beta=-0.34, p<0.001)$.

\section{Discussion}

In this cross-cultural study, we found a difference in educational level: the Dutch patients were more educated. This difference is in line with available data on educational level in these countries [35]. An important finding was the high rate of patients not working at the time of study participation. Even though we do not know why patients were not working, this confirms the high levels of functional impairment in CFS patients $[2,4]$. Our results show that this is even much more pronounced in Dutch patients. Apart from cultural differences between both countries, this difference can also be attributed to economical factors and differences in the health care and social security system. Another possible explanation is the significantly longer duration of chronic fatigue that was observed in the Dutch patients.

As expected, levels of fatigue severity were very high and the majority of the patients met a clinical level of CFS in both countries. In addition, physical and psychological QoL were poor in both samples, confirming earlier findings showing that high levels of disability are associated with CFS [2,3]. Fatigue severity and somatic complaints were significantly associated with lower physical QoL in both samples. In the Portuguese sample, psychological functioning was significantly lower, and depression and anxiety were significantly higher. About half of the Portuguese patients even met clinical levels of anxiety and depression. These results are in line with the cultural differences in the experience and expression of distress between both countries [24]. These important differences in psychological distress can also be explained by the fact that CFS is, in contrast to The Netherlands, not recognized as a specific disorder by primary care health professionals in Portugal. As a result, Portuguese patients may present with higher levels of frustration, anxiety, and feelings of loneliness, helplessness, and hopelessness [8].

The second study question regards the contribution of behavioral and cognitive variables to fatigue severity in both samples. The regression models explained $45 \%$ (Portugal) and $46 \%$ (Netherlands) of the variance in fatigue severity. Interestingly, there are important differences in the determinants that were significantly associated with fatigue severity. In relation to the behavioral determinants, higher levels of physical activity were significantly associated with lower levels of fatigue severity in the Portuguese sample, which is in accordance with previous research $[36,37]$ and strengthens the current recommendation for CFS patients to engage in physical activity rather than refraining from it [38]. Additionally, limiting behavior (reducing daily activities and excessive resting) was also significantly associated with fatigue severity in the Portuguese patients. The fact that Dutch patients were more physically active and that physical activity was not a significant determinant of fatigue severity in this sample backs the importance of tailored physical activity for CFS patients (e.g., Graded Exercise Therapy). However, finding a good balance between activity and rest on a daily basis is equally important $[18,33,38]$. This assumption is reinforced by the fact that a boom-and-bust (all-or-none behavior) cycle was 
significantly associated with higher fatigue severity levels in the Dutch patients, which is in line with previous research [13, 16]. With respect to illness representations, in the Portuguese sample, the belief that CFS is a very serious condition (identity) was significantly associated with higher fatigue severity levels. This result confirms earlier findings that patients who believe that their illness is very severe adopt more passive ways of dealing with their health problem (such as limiting behavior) and, as a result of that, present higher levels of disability and psychological distress [2, 13, 17]. In fact, emotional response was also significantly associated with fatigue severity in the Portuguese sample. Emotional response was a significant determinant only in the Portuguese sample, which is in line with existing differences in psychological distress between the two countries [24]. For Dutch patients, the perception of having personal control over the illness was significantly associated with lower fatigue levels. On the other hand, thinking that CFS has severe consequences was significantly associated with higher fatigue severity. Overall, our findings are in line with previous research in which positive illness beliefs are associated with lower fatigue severity, and negative beliefs with higher fatigue severity [13].

While these findings are valuable, several limitations of this study should be discussed. A major limitation is that it was carried out in health care centers and patients associations. As a consequence, the results cannot be generalized to the whole CFS population in both countries. In addition, there was a difference in recruitment strategy. Portuguese patients were partly recruited via medical doctors, while all Dutch patients were recruited via e-mail by the patient association only. This may have led to a selection bias. Moreover, due to the fact that the confirmation of the diagnosis of CFS was based on self reports, it cannot be excluded that some patients do not fulfill all the CDC criteria for CFS. Ideally, this diagnosis should also rule out other somatic and psychiatric causes of the symptoms, by means of a full clinical assessment. Differences between the two samples in depression, anxiety and psychological QoL, can thus also be attributed to differences in diagnosis. Another limitation is the cross-sectional design of the study which limits the conclusions that can be drawn about the causality of the relationships. A longitudinal study would provide more valid data on the psychological predictors of CFS and the relationship between fatigue severity and disability. Moreover, there were differences in the sample size, and only female patients were included in the analyses. Finally, due to the fact that there are no normative data for the Portuguese CIS-20R and the BSI, the comparisons of the respective clinical levels should be interpreted with care.

Despite these limitations, this study is innovative due to fact that cross-cultural research on psychological aspects of CFS is very limited. CFS is considered to be a multifactorial disorder, with biological, social, and psychological factors contributing to its onset and perpetuation. Our findings suggest that fatigue severity and related impairment are very similar in CFS patients from Portugal and The Netherlands, which is in line with the idea that the illness is not restricted to one type of culture $[22,23]$. Nevertheless, there seem to be significant differences in psychological distress between the Portuguese and the Dutch patients. This study shows that a psychological approach can contribute to the understanding and treatment of CFS. The fact that illness representations and behavioral patterns were important determinants of fatigue severity in both countries suggest that similar interventions, such as psychological interventions that address patients' beliefs about their illness as well as interventions that focus on behavioral regulation or modification strategies, should be offered internationally. However, due to the differences found in the specific determinants, interventions should also be tailored to patients' needs and focus more on emotion regulation and the increase of physical activity levels in Portuguese patients, while encouraging personal control and establishing a good balance between daily activities and rest can be important intervention targets for Dutch patients.

Acknowledgments This study was carried out as part of a grant from the Portuguese Foundation for Science and Technology (SFRH/BD/ 47579/2008). The authors would like to thank the patients that participated in this research, the medical doctors from the health care institutions, and the volunteers from the patients associations in both countries.

\section{References}

1. Kroenke K, Spitzer R, Williams JBW. The PHQ-15: validity of a new measure for evaluating the severity of somatic symptoms. Psychsom Med. 2002;64:258-66.

2. De Gucht V, Maes S. Explaining medically unexplained symptoms: toward a multidimensional theory-based approach to somatisation. J Psychosom Res. 2006;60:349-55. doi:10.1016/ j.jpsychores.2006.01.021.

3. Hardt J, Buchwald D, Wilks D, Sharpe M, Nix WA, Eagle UT. Health-related quality of life in patients with chronic fatigue syndrome: an international study. J Psychosom Res. 2001;51:431-4. doi:10.1016/S0022-3999(01)00220-3.

4. Afari N, Buchwald D. Chronic fatigue syndrome: a review. Am J Psych. 2003;160:221-36. doi:10.1176/appi.ajp. 160.2.221.

5. Fukuda K, Strauss SE, Hickie I, Sharpe MC, Dobbins JG, Komaroff A. The chronic fatigue syndrome: a comprehensive approach to its definition and study. Annals Int Med. 1994;121:953-9.

6. Prins JB, der Meer JWM V, Bleijenberg G. Chronic fatigue syndrome. Lancet. 2006;367:346-55. doi:1016/S0140-6736(06)6807.

7. Johnson SK. Depression and fatigue. In: DeLuca J, editor. Fatigue as a window to the brain. Cambridge: MIT; 2005. p. 157-72.

8. Lehman AM, Lehman DR, Hemphill KJ, Mandel DR, Cooper LM. Illness experience, depression and anxiety in chronic fatigue syndrome. J Psychosom Res. 2002;56:461-5. doi:10.1016/S00223999(02)00318-5.

9. Harvey SB, Wadsworth M, Wessely S, Hotopf M. The relationship between prior psychiatric disorder and chronic fatigue: evidence from a national birth cohort study. Psychol Med. 2008;38:933-40. doi:10.1017/S0033291707001900. 
10. Clark C, Goodwin L, Satnsfeld SA, Hotopf M, White PD. Premorbid risk markers for chronic fatigue syndrome in the 1958 British birth cohort. Br J Psych. 2011;199:323-9. doi:10.1192/ bjp.bp. 110.083956.

11. Allen LA, Escobar JL. Fatigue and somatization. In: DeLuca J, editor. Fatigue as a window to the brain. Cambridge: MIT; 2005. p. 173-83.

12. Knottnerus JA. Advisory report on chronic fatigue syndrome. Health Council of the Netherlands. 2005. http://www.gezondheidsraad.nl/ sites/default/files/05@02E.pdf. Accessed 12 Jan 2012.

13. Moss-Morris R. Symptom perceptions, illness beliefs and coping in chronic fatigue syndrome. J Ment Health. 2005;14:223-35. doi:10.1080/09638230500136548.

14. Leventhal H, Leventhal EA, Contrada RJ. Self regulation, health, and behavior: a perceptual-cognitive approach. Psychol Heal. 1998;13:717-33. doi:10.1080/08870449808407425.

15. Weinman J, Petrie KJ, Moss-Morris R, Home R. The illness perception questionnaire: a new method for assessing illness perceptions. Psychol Heal. 1996;11:431-46. doi:10.1080/08870449608400270.

16. Moss-Morris R, Spence MJ, Hou R. The pathway from glandular fever to chronic fatigue syndrome: can the cognitive behavioral model provide the map? Psychol Med. 2011;41:1099-107. doi:10.1017/S003329171000139X.

17. Heijmans MJWM. Coping and adaptive outcome in chronic fatigue syndrome: importance of illness cognitions. J Psychosom Res. 1998;45:39-51.

18. Graded exercise therapy: A self-help guide for those with chronic fatigue syndrome/myalgic encephalomyelitis. NHS Foundation Trust and St. Bartholomew's Hospital 2009. http://drleigh.org/ wp-content/uploads/2008/12/chronic-fatigue-exercise-programspecifics.pdf. Accessed 20 May 2009.

19. Fulcher KY, White PD. Strength and physiological response to exercise in patients with chronic fatigue syndrome. J Neurol Neurosurg Psych. 2000;69:302-7. doi:10.1136/jnnp. 69.3.302.

20. Price JR, Mitchell E, Tidy E, Hunot V. Cognitive behaviour therapy for chronic fatigue syndrome in adults. Cochrane Database Syst Rev. 2008;3:CD001027.

21. McGuire L, Edmonds L, Price JR. Exercise therapy for chronic fatigue syndrome. Cochrane Database Syst Rev. 2004;3:CD003200.

22. Hickie I, Davenport T, Vernon SD, Nisenbaum R, Reeves WC, HadziPavlovic D, et al. Are chronic fatigue and chronic fatigue syndrome valid clinical entities across countries and health-care settings? Aust $\mathrm{N}$ Z J Psych. 2009;43:25-35. doi:10.1080/00048670802534432.

23. Jo Cho H, Menezes PR, Hotopf M, Bhugra D, Wessley S. Comparative epidemiology of chronic fatigue syndrome in Brazilian and British primary care: prevalence and recognition. Br J Psych. 2009;194:117-22. doi:10.1192/bjp.bp.108.051813.
24. Eurobarometer. Mental Health. Special eurobarometer 345/Wave 73.2. European Comission; 2010.

25. Eurobarometer. Health and food. Special eurobarometer 334/ Wavw 72.3. European Comission; 2010.

26. Vercoulen JHMM, Alberets M, Bleijenberg G. De Checklist Individual Strenght (CIS). Gedragstherapie. 1999;32:31-6.

27. De Vree B, Van der Werf S, Prins J, Bazlmans E, Vercoulen J, Servaes P. Meetinstrumenten bij chronische vermoeidheid. Gedragstherapie. 2002;35:157-64.

28. Marques M, De Gucht V, Maes S, Leal I. Relação entre exercício físico e fadiga percebida. In: Maia A, Silva S, Pires T, editors. Proceedings of the I congress on health and behaviour of the Portuguese speaking countries. Braga: CIPSI Edições; 2009. p. $505-11$.

29. Degoratis L. Brief symptom inventory: administration, scoring and procedures manual. 3rd ed. Minneapolis: National Computer System;1993.

30. De Beurs E. Zitman F The Brief Symptom Inventory (BSI): de betrouwbaarheid en validiteit van een handzaam alternatief voor de SCL-90. Maandblad Geestelijke Volksgezondheid. 2005;61:120 41.

31. Ware JE, Kosinski M, Turner-Bowker DM, Gandek B. How to score version 2 of the SF-12 ${ }^{\circledR}$ Health Survey. Lincoln: Quality Metric Incorporated; 2002.

32. Wendel-Vos GCW, Schuit AJ, Saris WHM, Kromhout D. Reproducibility and relative validity of the short questionnaire to assess health-enhancing physical activity. J Clin Epidemiol. 2003;56:1163-9. doi:10.1016/S0895-4356(03)00220-8.

33. Spence M, Moss-Morris R, Chalder T. The behavioural responses to illness questionnaire (BRIQ): a new predictive measure of medically unexplained symptoms following acute infection. Psychol Med. 2005;35:583-93. doi:10.1017/S0033291704003484.

34. Broadbent E, Petrie KJ, Main J, Weinman J. The brief illness perception questionnaire. J Psychosom Res. 2006;60:631-7. doi:10.1016/j.jpsychores.2005.10.020.

35. Key data on education in Europe. European Comission; 2009. Brussels: Education, Audiovisual and Culture Executive Agency.

36. Fulcher KY, White PD. Randomised controlled trial of graded exercise in patients with the chronic fatigue syndrome. BMJ. 1997;314:1647-52. doi:10.1136/bmj.314.7095.1647.

37. Moss-Morris R, Sharon C, Tobin R, Baldi JC. A randomised controlled graded exercise trial for chronic fatigue syndrome: outcomes and mechanisms of change. J Health Psychol. 2005;10:245-59. doi:10.1177/1359105305049774.

38. National Institute for Health and Clinical Excellence. Guideline 53: chronic fatigue syndrome/myalgic encephalomyelitis (or encephalopathy). London; 2007. 